Cinémas

Revue d'études cinématographiques

Journal of Film Studies

\title{
De la musicalité avant toute chose...
}

\section{François Jost}

Volume 3, numéro 1, automne 1992

Cinéma et Musicalité

URI : https://id.erudit.org/iderudit/1001175ar

DOI : https://doi.org/10.7202/1001175ar

Aller au sommaire du numéro

Éditeur(s)

Cinémas

\section{ISSN}

1181-6945 (imprimé)

1705-6500 (numérique)

Découvrir la revue

Citer cet article

Jost, F. (1992). De la musicalité avant toute chose.... Cinémas, 3(1), 8-18. https://doi.org/10.7202/1001175ar

\section{Résumé de l'article}

Selon Goodman, la musique se définit essentiellement comme un système de notation qui délimite une classe d'exécutions exemplifiant la partition et la transformant en oeuvre. Le film, aussi bien en tant que structure narrative qu'en tant que structure audiovisuelle, est en revanche irréductible à un système de notations aussi fort que la musique. Dans ces conditions, que signifie l'introduction d'une pertinence musicale, la musicalité, dans le narratif? En quoi déplace-t-elle le statut d'oeuvre d'art du film? En quoi construit-elle un autre spectateur? Voilà quelques-unes des questions que l'article essaie ici de résoudre. 


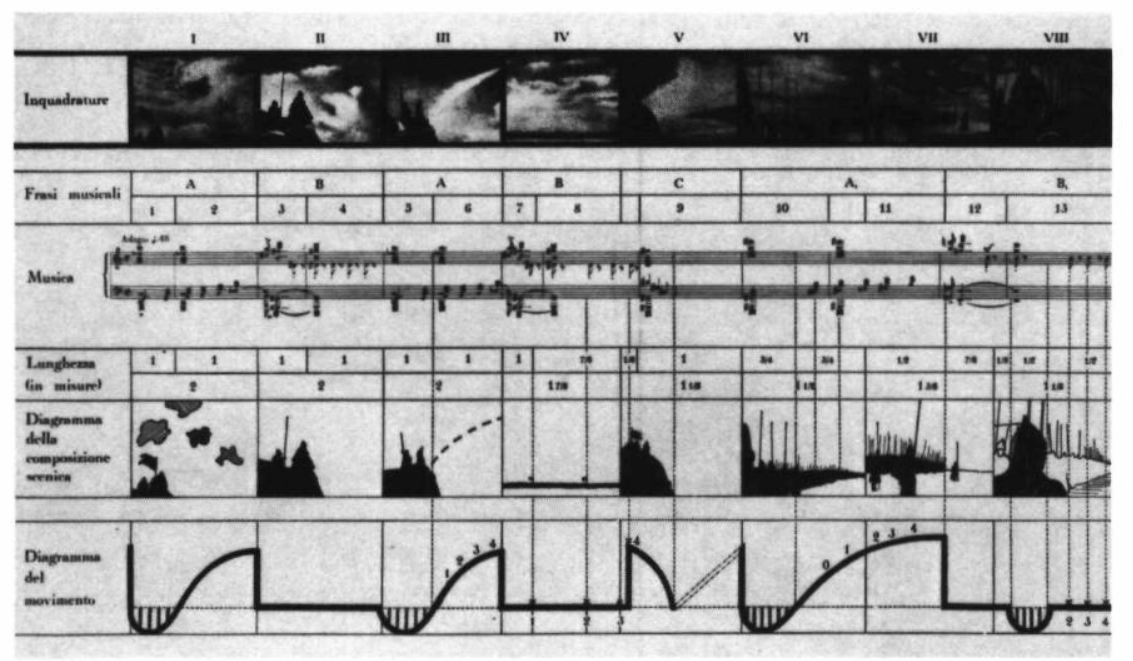

Alexandre Newski d'Eisenstein (1938)

Coll. Cinémathèque québécoise 


\title{
De la musicalité avant toute chose...
}

\section{François Jost}

\begin{abstract}
RÉSUMÉ
Selon Goodman, la musique se définit essentiellement comme un système de notation qui délimite une classe d'exécutions exemplifiant la partition et la transformant en cuvre. Le film, aussi bien en tant que structure narrative qu'en tant que structure audiovisuelle, est en revanche irréductible à un système de notations aussi fort que la musique. Dans ces conditions, que signifie l'introduction d'une pertinence musicale, la musicalité, dans le narratif? En quoi déplace-t-elle le statut d'œuvre d'art du film? En quoi construit-elle un autre spectateur? Voilà quelquesunes des questions que l'article essaie ici de résoudre.
\end{abstract}

\begin{abstract}
According to Goodman, music is essentially defined as a system of notation that bounds a class of executions exemplifying the score and transforming it into performance. In contrast, film, both as narrative structure and as audiovisual structure, cannot be reduced to a system of notation as powerful as that of music. Under these conditions, what is the meaning of the introduction of musical relevance, of musicality, into the narrative? How does this affect the status of the film as a work of art? How does it alter the way the spectator is constituted? These are the questions that this article attempts to resolve.
\end{abstract}

\section{La musique comme système notationnel}

Réfléchissant sur la question de l'authenticité de l'œuvre d'art, Nelson Goodman est amené à distinguer entre les œuvres autographiques et les œuvres allographiques. Tandis que, pour les 
premières, la distinction entre l'original et une contrefaçon a un sens, pour les secondes, elle n'en a aucun. Autant il est possible, par exemple, de copier un tableau de Vermeer, on ne peut copier la Symphonie fantastique sans refaire, du même coup, l'œuvre de Berlioz. Pour la musique, on peut certes contrefaire des exécutions (faire passer, par exemple, un disque pour un enregistrement inédit de Gould), mais l'exécution en elle-même, quelle que soit sa qualité, ne saurait être considérée comme une contrefaçon de l'œuvre originale.

Ce statut de la musique tient au fait qu'elle repose sur un système notationnel : la partition. Qu'est-ce qu'une partition? C'est, répond le philosophe américain, cette «autorité qui identifie une œuvre, d'exécution en exécution» (p. 166). En d'autres termes, la partition définit l'œuvre musicale en déterminant la classe d'exécutions qui lui appartiennent (y compris lorsqu'elle prévoit des parcours facultatifs, comme chez certains compositeurs modernes). Inversement, elle est déterminée de façon unique par le système notationnel qui la régit. Cette relation de la partition à «l'exemple» qu'en représente son exécution s'oppose à celle qu'entretient l'esquisse avec l'œuvre finie. Si celle-là peut contenir en germe le tableau à venir, elle ne «définit» pas une œuvre, au sens où elle délimiterait une classe d'exécutions, elle est une œuvre, dans la mesure où elle ne joue pas comme caractère dans un système notationnel.

Néanmoins, tout dans la partition musicale n'est pas notationnel. Le langage verbal des tempos - notamment, des termes comme presto, allegro, allegro vivace, moderato, etc. - affecte certes la qualité de l'exécution, mais «aucun manquement au tempo indiqué ne disqualifie une exécution d'être un exemple» (Goodman, p. 225).

Le «script» tient une position intermédiaire entre la partition et l'esquisse, puisqu'il fonctionne bien dans un système de notation (la langue), tout en étant l'œuvre elle-même. La répétition à l'identique d'un texte, quelle que soit l'édition, suffit à assurer la permanence de l'œuvre littéraire, mais cette répétition ne saurait s'identifier à une classe d'exécution puisque celle-ci définit des exemples de l'œuvre sans être l'œuvre elle-même1. Et Goodman conclut :

En somme, un art établi devient allographique seulement lorsque la classification des objets ou des événements en cuvre est projetée légitimement à partir d'unc classification antérieure et est complètement définic, indépendamment du procès de production, dans les termes d'un systèmc notationnel (p. 237). 
Si l'on en vient à des arts scéniques, on ne peut que constater, une fois de plus, leur très grande hétérogénéité en tant qu'œuvre. Le texte d'une pièce de théâtre est, en ce qui concerne les dialogues, assimilable à une partition : quelle que soit la façon dont on dit Le Médecin malgré lui, ce sera toujours la pièce de Molière; en ce qui concerne les didascalies, en revanche, il est du côté du script : aucune indication scénique, aucun décor, aucune mise en scène n'est susceptible d'assurer la répétition à l'identique d'une représentation théâtrale. Ces remarques valent évidemment aussi pour le cinéma :

Un script de cinéma pour un film muet n'est ni l'œuvre filmique ni une partition pour l'exécuter mais, bien qu'on l'utilise dans le tournage d'un film, il est sous d'autres rapports dans une relation à l'œuvre aussi vague qu'unc description verbale d'une peinture à la peinture elle-même (Goodman, p. 250).

Allons plus loin et tentons de préciser le statut du film. À première vue, l'image cinématographique n'est pas reproductible : aucun cadrage, aucun mouvement de caméra, non plus qu'aucune empreinte du réel ne peuvent être refaits et, à partir d'un même scénario, on peut tourner une quantité indéfinie d'œuvres différentes. Tout semble donc situer le film muet du côté de l'autographique. Que l'on puisse multiplier les copies à partir d'un négatif original n'y change rien. Le fait que le cinéma est un art à deux phases n'altère nullement sa nature autographique : de même que les différentes gravures obtenues à partir d'un original sont des exemples de cet original, contrairement à tout autre image produite autrement que par impression (imitation ou contrefaçon), la copie de film ne mérite ce nom que parce qu'elle est un produit terminal fait à l'image de l'original et non parce qu'elle en serait une contrefaçon. On voit bien, parvenu en ce point, ce qui écarte le film de la musique. Alors que celle-ci repose sur un système de notation qui délimite une classe d'exécutions, celui-là ne peut être assimilé à une partition ni du point de vue de l'image ni du point de vue du scénario (conçu comme organisation narrative).

Je sens néanmoins, chez le lecteur avisé, l'envie de m'interrompre. Peut-on traiter toutes les phases de la réalisation de la même manière? Sans doute pas. Le montage, en particulier, n'est-il pas une opération proprement allographique, puisqu'il suffit, après tout, d'appliquer quelques règles arithmétiques à la copie de travail, pour refaire un même film, ou au négatif, pour conformer la copie de travail à son original? 


\section{L'intention auctoriale}

Lorsque Eisenstein définit le «montage métrique», il pense la bande-image selon le modèle de la musique. En effet, le critère qui permet de le définir est la longueur du morceau monté, déterminée par une «formule» que le cinéaste apparente à la mesure en musique et qui imprime un rythme quasi mécanique et reproductible à une action, grâce à l'application d'une proportion $(1 / 2,1 / 3,1 / 4)$, dotée, en tant que telle, d'un équivalent musical.

Le montage rythmique, en revanche, rompt avec cette logique purement notationnelle, et prend en considération la nature proprement autographique de l'image cinématographique, puisqu'il ne s'agit plus d'appliquer une formule quasi mathématique, mais de moduler la longueur des plans en fonction de ce qu'ils donnent à voir et, notamment, des mouvements qu'ils reconstituent. Ainsi, nous dit le cinéaste russe, pour avoir voulu plier mécaniquement le rythme complexe du visuel, la séquence de la danse religieuse des masques dans Tempête sur l'Asie (Poudovkine, 1929) est ratée.

Néanmoins, la volonté d'introduire une rationalité notationnelle dans l'image anime très fortement la théorie du montage d'Eisenstein. Le montage tonal, comme le montage harmonique, bien qu'ils soient apparemment fondés sur un concept, la tonalité, en apparence beaucoup plus labile, obéissent en fait à des critères objectivables et formalisables : cette «résonance émotionnelle d'un plan» qu'est la tonalité n'est pas évaluée de façon purement impressionniste, elle est tout aussi mesurable que la longueur d'un plan. Par exemple, «si nous attribuons à un morceau quelconque une appellation comparative et émotionnelle comme "plus sombre", nous pouvons trouver tout aussi bien pour ce morceau un coefficient mathématique représentant son degré d'éclairage» (Eisenstein, p. 66). Notée, en tous les sens du terme, l'image devient musique : les vibrations lumineuses optiques (faites de brouillard et de luminosité) révèlent une «analogie complète» avec le mode mineur. Comme on sait, Eisenstein ira encore plus loin dans cette recherche des correspondances, puisque la tâche du compositeur de musique sera d'abord d'analyser le mouvement visuel contenu dans le plan, puis dans la suite de plans offerts par le montage, pour arriver, finalement, à la «coïncidence du mouvement de la musique avec celui du contour visuel» (p. 317). Et le cinéaste russe de se lancer dans l'analyse de multiples séquences fondées sur une coïncidence absolue entre le mouvement de la musique et celui du regard, illustrant sa théorie grâce à des quasi-projections des portées musicales sur la structure plastique de l'image (pp. 325 s.) : 


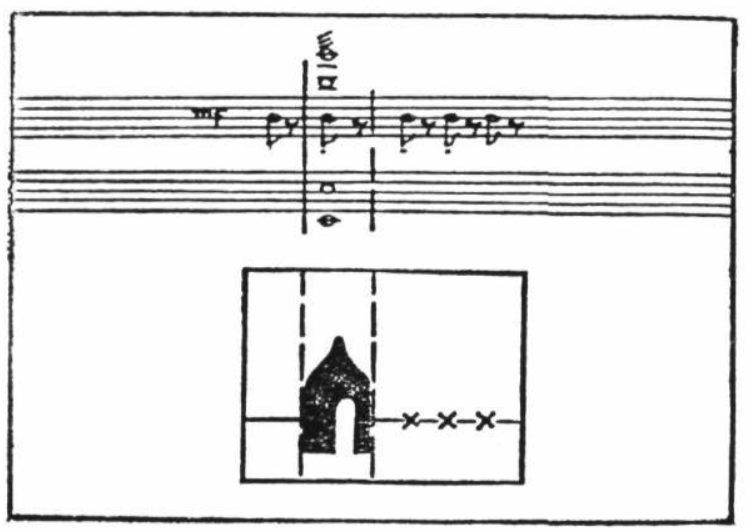

Chez Eisenstein, on le voit, c'est bien la musique comme système notationnel qui devient un modèle ${ }^{2}$. Certes, on peut se débarrasser d'un coup de plume d'une telle conception en contestant la valeur notationnelle des diagrammes du maître soviétique. Ils sont plus proches des «graphismes musicaux» que de la notation précise $^{3}$, plus proche de l'esquisse que de la partition. En effet, il est évident que, si on repartait de telles schématisations pour refaire l'image, on aboutirait à un résultat qui, pour garder le même mouvement, n'épouserait cependant pas les contours visuels du plan d'Alexandre Newski. Toute image tournée a posteriori serait donc comme un dessin ou une peinture faite d'après une esquisse et non d'après l'exécution d'une partition.

Légitime du point de vue de la théorie goodmanienne, cette objection n'annihile évidemment pas l'objection ni la recherche d'un modèle fondé sur la musique, rêve d'un œil musical qui suivrait dans l'image les chemins tracés par la partition. Même si l'on doit la ranger au rang des illusions synesthésiques, la musique participe des intentions auctoriales qui donnent forme à l'œuvre cinématographique. L'objection tombe complètement quand l'auteur se détourne du figuratif. Prenez Arnulf Rainer (19571960), de Kubelka. Ce film fait alterner des photogrammes blancs et des photogrammes noirs, accompagnés ou non d'un son blanc, selon un calcul rythmique précis. "Cinéma-musique» par son désir de ne pas signifier, selon Dominique Noguez (p. 93), Arnulf 
Rainer l'est aussi par son statut d'œuvre : en un sens, ce que nous voyons sur l'écran - une alternance de blanc et de noir - n'est que l'exécution d'une partition que chacun peut rejouer à sa manière, au cinéma ou à son domicile, dès lors qu'il possède la formule arithmétique structurant ce montage strictement métrique. Le film de Kubelka est la première œuvre allographique du cinéma, et peut-être... la seule possible.

Entre ces deux cas extrêmes du figuratif conçu comme système notationnel et de l'iconique réduit à un algorithme, il existe une conception plus lâche de la redondance informationnelle de l'image et du son, qui consiste à ne s'intéresser qu'aux correspondances, pour ainsi dire, verticales ou horizontales. La première se joue de la synchronie en substituant une note à un bruit : c'est ce que l'on voit dans les clips lorsqu'à une percussion de batterie correspond l'image d'une gifle; la seconde musicalise l'image par une sorte de synesthésie sauvage, fondée elle aussi sur la coïncidence temporelle, consistant, par exemple, à associer des mesures d'une musique globalement horizontale à un espace dénué de repères (on a reconnu Le Grand Bleu). Ici, la synchronie est au service du sémantique : la simultanéité de l'attaque sonore et du début d'une séquence fait glisser les prédicats attribués à l'ivresse des profondeurs marines vers la caractérisation de la musique, confirmant cette assertion goodmanienne : «Tout aspect de l'image peut participer à l'exemplification d'une couleur ou à l'expression d'un son» (p. 278).

Hormis des cas aussi visibles, toute la difficulté, pour le spectateur, est de rencontrer l'intention auctoriale.

\section{Attention spectatorielle et musicalité}

Les psycholinguistes distinguent entre «mémoire de situation» et «mémoire du texte». Alors que la première ne retient que la teneur des propositions et non des formulations précises, la seconde, à l'inverse, mémorise la lettre même, comme c'est le cas dans le «par cœur», les prières, etc. Or, curieusement, les spécialistes de la psychologie cognitive ne constatent guère de différence entre l'écoute musicale et les autres actes perceptifs : ici comme là, le sujet procéderait par schématisation; comme le récit, qui est compris grâce à l'identification d'indices renvoyant le lecteur ou le spectateur à des situations déjà connues, le morceau de musique, grâce à la répétition, donnerait à l'auditeur :

(...) la possibilité d'accéder el de récupérer les traces stockées — des indices - qui vont lui permettre, par les réitérations qu'elles subissent, d'organiser des relations dans son écoute présente, voire de la connecter à des schémas antérieurs (Deliège, p. 34) 4 . 
La répétition aurait le même rôle que dans la compréhension du récit et la maîtrise de l'écoute passerait par la maîtrise de la syntaxe.

De même qu'on peut reprocher à la sémiologie du cinéma d'avoir longtemps raisonné sur un spectateur moyen introuvable, on peut se demander quel auditeur pense la psychologie cognitive : en tentant de théoriser ce que font «la plupart des gens» (Clynes, p. 3 ), on néglige évidemment le fossé qui sépare l'auditeur occasionnel de musique classique du véritable mélomane, tout en supposant qu'ils situent les indices au même niveau. Par ailleurs, il va de soi que ces indices ne sont pas de même nature pour tous les morceaux : si la musique classique suit des règles strictes qui permettent d'identifier les thèmes, leurs répétitions ou leurs imitations, si la musique moderne en crée d'autres moins prévisibles, ces indices sont des configurations, des formes, dénuées de sens, quand bien même ils sémantisent le morceau (comme les motifs wagnériens ou bergiens).

En rapprochant compréhension du récit et écoute musicale, les psychologues de la musique ne théorisent donc que la perception d'un spectateur-auditeur qui fait l'apprentissage d'un morceau. À n'en pas douter, cette expérience est pourtant bien différente de celle du mélomane ou du musicien qui connaît a priori les formes, les thèmes ou les mélodies ou, même, qui découvre un nouveau morceau.

L'écoute musicienne ne se distingue-t-elle pas de l'écoute musicale par le fait que, loin d'identifier globalement des configurations, des thèmes, comme le dit Minsky, elle retient des notes, des mesures, en bref : la partition? Ce type d'écoute est, lui, très différent de la réception narrativisante. L'image figurative, en effet, renvoie immédiatement le spectateur à des indices narratifs et, par là même, à des situations narratives. L'image fait écran à la musicalité du visible et il va de soi qu'elle se prête plus à une mémoire de situation qu'à une mémoire de texte.

Selon Dan Sperber et Deirdre Wilson, la cognition humaine repose sur le fait que l'individu cherche d'abord à améliorer sa connaissance du monde. C'est ce principe de l'efficacité cognitive qui définit la pertinence :

(...)une information pertinente pour un individu est une information qui modifie et améliore la représentation globale que cet individu a du monde (p. 111).

On voit sans mal comment une telle conception s'applique presque textuellement au spectateur du film narratif : seules comptent pour lui, dans la réception du récit, les informations qui l'aident à 
construire et à comprendre le monde fictionnel. Et, pour cela, la mémoire de situation lui suffit bien.

Si l'on glisse facilement du film au monde, ce n'est pas seulement que l'un est construit sur le modèle de l'autre, c'est aussi que nous construisons l'un et l'autre de la même façon. Or, sollicitant la mémoire de texte, l'écoute musicienne d'un film est une lutte continuelle contre ce principe de pertinence qui régit notre communication verbale quotidienne. À l'inférence qui efface la lettre, elle oppose la matérialité de l'image ou des sons. En un sens, Mallarmé ne disait pas autre chose quand il brocardait ces usages du langage, «narrer, enseigner, même décrire», qui le réduisent à "cette fonction de numéraire facile et représentatif», assurant la circulation des idées comme une pièce de monnaie. Valéry, de son côté, soulignait que, «dans les emplois pratiques ou didactiques du langage, "la forme, c'est-à-dire la physique, le sensible, et l'acte même du discours ne se conserve pas; elle ne survit pas à la compréhension; elle se dissout dans la clarté; elle a agi; elle a fait son office"» (Escal, p. 138).

En mettant en avant la métaphore du langage cinématographique, la sémiologie du cinéma a, dès l'origine, développé malgré elle l'idée d'une image «numéraire». Comprendre un film, pour elle, c'est d'abord comprendre son déroulement narratif. Comme si celui-ci n'était que l'instrument d'une communication narrative.

Or, de même que l'auteur, en faisant un film, peut avoir d'autres intentions que de raconter une histoire, il arrive, plus souvent que ne le laissent entendre les théoriciens du cinéma, que l'on refuse cette attention continue à l'anecdote. Au spectateur narrativisant, qui reçoit globalement des situations, qui infère des significations du rapprochement des plans ou des séquences, il faut opposer le spectateur-musicien, amoureux du détail, soucieux de la répétition, inventeur de formes proprement invisibles.

La musicalité dans le film peut prendre diverses voies :

1. La "désémantisation" de l'image cinématographique. Cette entreprise, celle des années vingt et du cinéma expérimental, vise à mimer la nature fondamentalement monofaciale du signe musical (Escal, p. 133) en diluant la signification visuelle dans le montage, le trucage ou l'abstraction.

2. La décomposition du visible en thèmes et en motifs. La variation sur un thème est l'un des principes clés de la musicalité, mais elle s'oppose à la logique narrative pour laquelle la redondance de l'information ne peut être qu'au service de l'amélioration de la connaissance du monde de la fiction et, par là même, de la compréhension du récit. Si la variation est manifestement au cœur d'un film comme Je t'aime, je t'aime 
(Ridder qui sort de l'eau) ou de Providence (cf. le décor de la villa au bord de la mer), elle est plus ou moins perceptible selon qu'elle affecte le visible ou une thématique sous-jacente, selon qu'elle s'intègre à la diégèse ou non. Dans la mesure où rien n'incite, dans le paratexte filmique, à recevoir le film selon une pertinence musicale, il appartient au spectateur d'abord d'identifier ce qui peut faire thème (une configuration spatiale, un assemblage de sèmes ou un mouvement de caméra : je pense à cette entrée et à cette sortie d'Arkadin dans la maison de Zouk, qui se répondent à près d'une heure et demie de distance). De telles variations n'ont de sens que pour une mémoire de texte, qui retient le plan, sa disposition ou les mouvements qui le composent.

3. La musicalisation du visible. Ce dernier stade de la musicalité réside évidemment dans la conformation du complexe images-sons à une forme importée de la musique. Le fait que la sémiologie du cinéma a choisi la pertinence narrative et langagière explique en partie que les syntagmes soient pensés comme des unités indépendantes du texte : forme un tout ce qui répond à une unité de situation ou de signification (chronologique ou achronologique). Or, de nombreux films, à toutes les époques de l'histoire du cinéma, présentent des suites de plans dont l'unité n'existe que dans le renvoi thématique à la globalité de l'œuvre. Au hasard et dans le désordre : les visages de Martha dans La Ligne générale, le motif des diagonales dans Un chien andalou ou le prologue de The Draugthman's Contract, où une suite de saynètes discontinues esquissent par touches successives le thème narratif principal. De même que la succession des notes et des silences d'un morceau de musique obéit à une logique d'ensemble qui est celle de la forme musicale, ces plans ne font syntagmes que si on les rapporte à l'ensemble du film conçu comme une composition. Je ne reviendrai pas sur les analyses que j'ai menées il y a une quinzaine d'années, seul ou avec Dominique Château, et qui révélaient l'existence de séquences narratives construites en miroir ${ }^{5}$. Je me contenterai de remarquer que ce type de structure, directement hérité de la musique (il est des fugues de Bach en miroir, comme des passages de Wozzeck), est vraisemblablement responsable du rejet critique qu'aura pu engendrer une certaine modernité des années 60 et, vingt ou trente ans plus tard, un cinéaste comme Greenaway. Traiter le narratif et le représentatif comme une forme pure apparaît encore comme une légèreté ou une indifférence coupables.

Il ne suffit pas de mettre au point un système de notation visuelle pour assurer l'interprétation musicale d'un film. Pour exister, celle-ci doit aussi rencontrer l'attention spectatorielle. Ici comme ailleurs, le statut de l'œuvre cinématographique ne naît que de la 
rencontre des ces deux mouvements : intention et attention. Une chose est sûre, en effet : quelles que soient les voies qu'emprunte celui qui fait le film et celui qui le voit, il s'agit toujours, en fin de compte, d'aller chercher auprès d'un art légitime de quoi fonder le statut artistique du cinéma, afin de faire ou de voir le film comme une œuvre. Ce n'est pas là le moindre mérite de la musicalité au cinéma.

\section{NOTES}

1 «Ce que produit l'écrivain est ultime; le texte n'est pas un simple moyen en vue de lectures orales à la manière dont une partition est un moyen en vue d'exécutions de musique.» Propos de Nelson Goodman, Langages de l'art (Nîmes : Chambon, 1990).

2 Marie-Dominique Popelard évoque aussi l'exemple d'Eisenstein dans «Philosophie analytique et analyse musicale : invitation à quelque travail en commun", Revue Analyse Musicale 28 (juin 1992).

3 Selon la classification d'Erhardt Karkoschka, Das Schriftsbild der Neuen Musik (Celle : Hermann Mock, 1966).pp. 19 s. Cité par Nelson Goodman.

4 Mon article emprunte à la bibliographic très complète de l'article d'Irène Deliège, «De l'activité perceptive à la représentation mentale de l'œuvre musicale», Analyse Musicale 28 (juin 1992).

5 Cf. notamment, Nouveau cinéma, nouvelle sémiologie (Paris : U.G.E., 1979, repris en 1983 par les éd. de Minuit).

\section{OUVRAGES CITÉS}

Clynes, M. (ed). Music, Mind and Brain. New York : Plenum Press, 1982.

Deliège, Irène. "De l'activité perceptive à la représentation mentale de l'œuvre musicale». Revue Analyse Musicale 28 (juin 1992) pp. 29-35.

Eisenstein, Serge. Le Film : sa forme, son sens. Paris : Bourgois, 1976.

Escal, François. Contrepoints, Musique et littérature. Paris : Méridiens Klincksieck, 1990.

Goodman, Nelson. Langages de l'art. Nîmes : Chambon, 1990.

Noguez, Dominique. Éloge du cinéma expérimental. Paris : Centre Georges Pompidou, 1979.

Sperber, Dan et Deirdre Wilson. La Pertinence. Paris : Minuit, 1989. 